\title{
Optical coding of SPAD array and its application in compressive depth and transient imaging
}

Sun, Qilin, Dun, Xiong, Peng, Yifan, Heidrich, Wolfgang

Qilin Sun, Xiong Dun, Yifan Peng, Wolfgang Heidrich, "Optical coding of SPAD array and its application in compressive depth and transient imaging," Proc. SPIE 11187, Optoelectronic Imaging and Multimedia Technology VI, 111870D (18 November 2019); doi: 10.1117/12.2538783

SPIE. Event: SPIE/COS Photonics Asia, 2019, Hangzhou, China 


\title{
Optical coding of SPAD array and its application in compressive depth and transient imaging
}

\author{
Qilin Sun ${ }^{\mathrm{a}}$, Xiong Dun ${ }^{\mathrm{b}}$, Yifan Peng ${ }^{\mathrm{c}}$, and Wolfgang Heidrich ${ }^{\mathrm{a}}$ \\ ${ }^{a}$ King Abudullah University of Science and Technology, Thuwal, Saudi Arabia \\ ${ }^{b}$ Tongji University, Shanghai, China \\ 'Stanford University, Stanford, CA, USA
}

\begin{abstract}
Time-of-flight depth imaging and transient imaging are two imaging modalities that have recently received a lot of interest. Despite much research, existing hardware systems are limited either in terms of temporal resolution or are prohibitively expensive. Arrays of Single Photon Avalanche Diodes (SPADs) are promising candidates to fill this gap by providing higher temporal resolution at an affordable cost. Unfortunately, state-of-the-art SPAD arrays are only available in relatively small resolutions and low fill-factor. Furthermore, the low fill-factor issue leads to more ill-posed problems when seeking to realize the super-resolution imaging with SPAD array.

In this work, we target on hand-crafting the optical structure of SPAD array to enable the super-resolution design of SPAD array. We particularly investigate the scenario of optical coding for SPAD array, including the improvement of fill-factor of SPAD array by assembling microstructures and the direct light modulation using a diffractive optical element. A part of the design work has been applied in our recent advance, where here we show several applications in depth and transient imaging.
\end{abstract}

Keywords: low fill-factor, diffractive optical element (DOE), end-to-end design, compressive sensing, time-offlight/transient imaging

\section{INTRODUCTION}

Enabling an array of single-photon level detectors with high time and spatial resolution is of high interest, no matter in scientific research or commercial application. However, a solution meeting these goals is not yet available to either academia or industry. Generally, capturing ultrafast process like light-in-flight ${ }^{1}$ requires singlephoton level sensitivity with high temporal resolution and large field-of-view imaging capability, that boost the emergence and application of SPAD array. Limited by the low resolution of a SPAD array, much research work has attempted to reconstruct the high-resolution transient or depth images by shifting a 2D SPAD array with a fixed lens ${ }^{2}$ or employing a galvo mirror to scan a 1D line SPAD camera. ${ }^{3}$

Standard CMOS fabrication process shows significant advantages regarding the routine of on-chip integration with the electronics required for photon-counting and photon-timing techniques. However, the commercial inpixel circuitry designing of $\mathrm{SPAD}^{4-6}$ has to compromise the proportion of detector active area. That said, the low fill-factor of SPAD not only trades off the sensitivity but also makes the sampled signal aliased and more ill-conditioned for a super-resolution post-processing.

Therefore, we seek to investigate the optical coding that not only maintains the advantages of SPAD array the ultra-high temporal resolution and the single photon level sensitivity but also enhances its spatial sampling performance. Due to optical coding, improving its spatial resolution becomes viable. To obtain a better sampling or anti-aliasing behavior, we seek to improve the fill-factor of SPAD array ${ }^{7}$ and design an anti-aliasing filter in an end-to-end manner for a super-resolution target. To increase the fill-factor of circuit-on-board SPAD array, a variety of approaches have been implemented, such as refractive ${ }^{8,9}$ and diffractive ${ }^{10}$ microlens array and reflective-based structures. ${ }^{11}$

Further author information: (Send correspondence to Qilin Sun)

Qilin Sun: qilin.sun@kaust.edu.sa; Xiong Dun: dunxiong@gmail.com; Yifan Peng: evanpeng@stanford.edu

Optoelectronic Imaging and Multimedia Technology VI, edited by Qionghai Dai, Tsutomu Shimura, Zhenrong Zheng, Proc. of SPIE Vol. 11187, 111870D · C 2019 SPIE · CCC code: 0277-786X/19/\$21 - doi: 10.1117/12.2538783 
With the fill-factor improved SPAD array, compressive sensing (CS), which is particularly beneficial to those cases where incorporating an array of sensors is infeasible (e.g., the SPAD array), becomes a feasible approach to improve its spatial resolution. Recently, a low-budget but high-resolution short-wave infrared camera has been realized $^{12}$ by combining a low-resolution SWIR camera $(64 \times 64)$ with a digital micromirror device $(\mathrm{DMD})$. This combination inspires the use of CS approach together with SPAD array to enable a realistic high spatial resolution. ${ }^{7}$

In addition, motivated by recent advances in end-to-end optimization, ${ }^{13}$ more complicated phase modulation elements have been reported. For instance, full end-to-end pipelines have been applied in the design of depthencoding PSFs for shape-from-defocus applications. ${ }^{14,15}$

In this work, we design and fabricate different kinds of microstructures to improve the fill-factor of SPAD array, including refractive and diffractive-based microlens array, as well as reflective-based structures. In addition, we design and fabricate an anti-aliasing filter that can be directly placed in front of a conventional low-fill factor SPAD camera. Finally, we apply the optical design to the compressive sensing based scenario to reconstruct high-resolution intensity imaging, depth, and transient imaging.

\section{OPTICAL CODING OF SPAD ARRAY}

\subsection{Design of micro concentrators}

Note, that each unit on our bare SPAD chip, whose size is $150 \mu \mathrm{m} \times 150 \mu \mathrm{m}$, has only a $30 \mu \mathrm{m}$ diameter's active area. That said, the fill-factor of the SPAD array is $3.14 \%$ only, making the compressive sensing setup ill-conditioned. It is demanding to improve its fill-factor to enable the ability of supporting CS methods and improve the light efficiency.

\subsubsection{Design of microlens arrays (MLAs)}

To mitigate the low fill-factor issue, we design different kinds of MLAs, including refractive MLA (RMLA), $2 \pi$ period diffractive MLA ${ }^{10}$ (DMLA) and $4 \pi$ period DMLA.

The refractive microlens array is designed as a non-spherical lens optimized for the refractive index of IPS ${ }^{16}$ by Zemax. The $2 \pi$ period DMLA is designed as a standard Fresnel lens array at $655 \mathrm{~nm}$ with a focal length of $1.035 \mathrm{~mm}$. The $4 \pi$ period DMLA is obtained by a modulo $4 \pi$ zone plates for $655 \mathrm{~nm}$ from the refractive lens. All of our MLAs have no dead zone between microlenses for square pixels.

\subsubsection{Design of reflective based micro concentrators}

We design the reflective based structures, including compound parabolic concentrators $(\mathrm{CPC})^{11,17,18}$ and our proposed trunked rectangular concentrators with micro lens (TRCM). Note, thet there exist alternative kinds of reflective based micro concentrators like trucked-con (TCC) and plain paraboloid (PPC). However, their performance has been proven worse ${ }^{11}$ than that of CPC.

After installing the glass window for the bare SPAD array, we measure the distance between the glass window surface and the sensor surface. According to the measured data, we design the microlens arrays with a focal length of $1.035 \mathrm{~mm}$.

\subsubsection{End-to-end design of anti-aliasing filter}

Instead of directly improving the fill-factor and collecting the light over the pixel block, employing an end-to-end design manner can be valuable. To this point, we aim to learn an optimal anti-aliasing filter that can be directly placed in front of a commercially available SPAD array without taking risks of crafting the sensor. Joint with a deep reconstruction network, we learn a PSF that provides the optimal sampling for the current sensor structure. We initially set the front focal plane as the pupil of the whole optical system and design a phase mask over there. Therefore, the relation between the phase mask and the sensor plane becomes a simple Fourier transform. After training and obtaining the target PSF, we use the GS-based phase retrieval method ${ }^{19}$ to retrieve the height map of DOE. This is a part of our on-going work. 
a
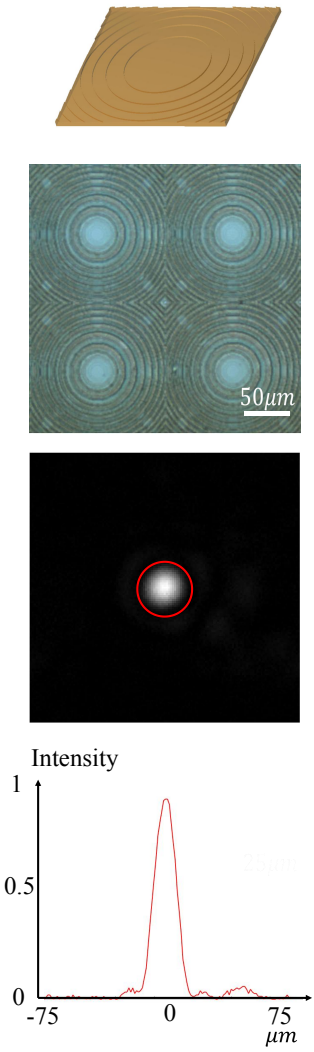

b
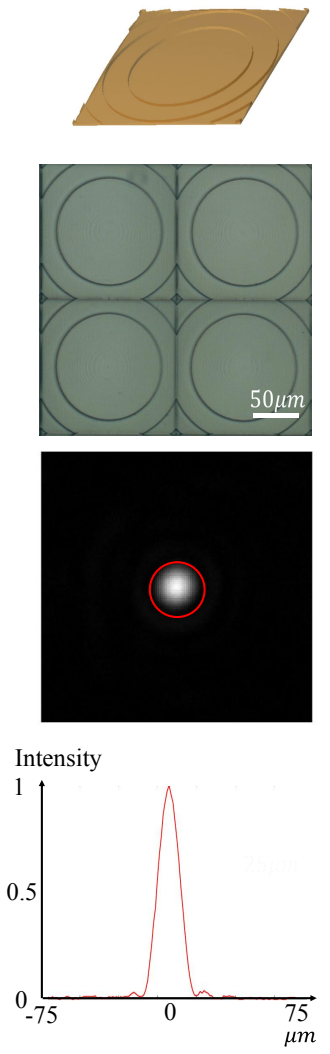

$\mathrm{C}$
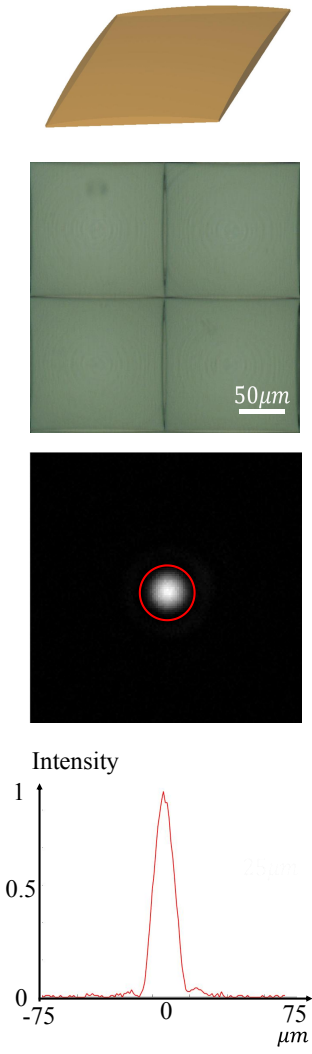

$\mathrm{e}$
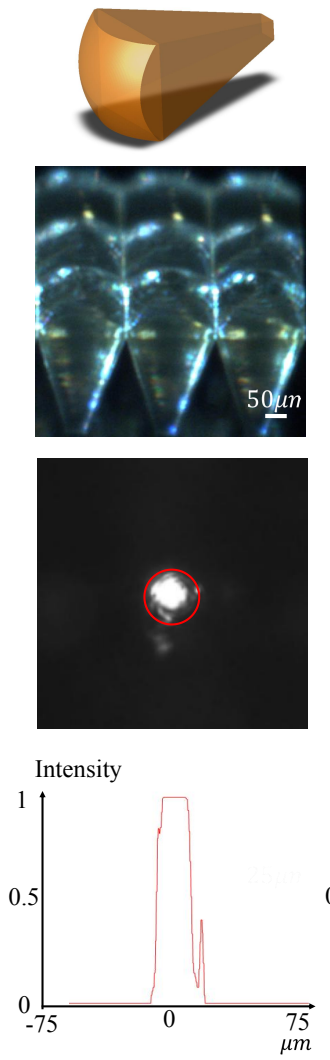
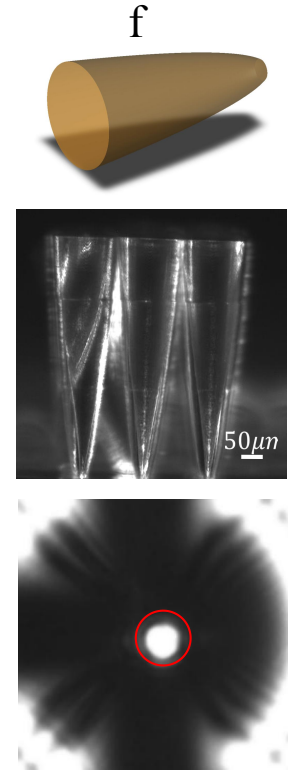

Intensity

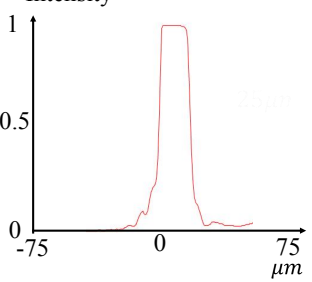

Figure 1. Optical (a, b, c) and electrical microscopy images, and focal spots with profiles recorded. (a) Diffractive microlens array with $2^{4}$ phase levels structure. (b) Diffractive microlens array with $4 \pi$ period. (c) Refractive microlens array. We take it as a reference. (d) Our proposed TRCM. (e) CPC. We take it as a reference. All profiles are normalized by its maximum value.

\subsection{Fabrication and analysis}

Figure 1 shows the 3D models, microscopy images, focal spots, and its profiles of those micro concentrators.

The lenses presented in Figure 1a and the end-to-end designed phase mask are fabricated by direct laser writing with SU-8 photoresist and etched the pattern onto the silica wafer by RIE etching. Similar to conventional 3D fabrication processing, $2 \pi$ DMLA consisting of 16 phase levels requires four successive binary masks $\left(16=2^{4}\right)$, further resulting in a fact that the alignment between layers can be challenging. The illustration of alignment error and mixing is shown in Figure 1a. The measured efficiency of this MLA is $52.87 \%$ under collimated illumination. The advantage of this method is that one can etch the SU-8 photoresist onto a silica wafer and enable a more stable pattern. However, the most significant shortcoming of this method compared with the above two lies on a fact that the essential low diffractive efficiency may result in non-trivial interference between neighboring pixels.

All other micro-structures are fabricated by Nanoscribe using IP-S photoresist. Nanoscribe is a 3D printer that allows for producing almost arbitrary micro-shapes with optically smooth surfaces in an additive and toolfree way. IP-S is a medium-resolution photoresist for smooth surfaces and fast structuring of large 3D parts.

\subsection{Performance analysis}

We analyze the efficiency through Zemax simulation. Figure 2 shows the simulated results of all refractive-based structures with considering the installation air gap (we set $2 \mu \mathrm{m}$ as an example here) and MLAs at its focal plane. Here, for DMLAs, we only show the result of $2 \pi$ DMLA as the efficiency simulated is quite similar for the 
designed wavelength. We observe our proposed TRCM outperforms that of other structures from f/2 to f/20. In contrast, the MLAs only has a low concentrator around 10 even at $f / 20$. To this end, we conclude that our proposed TRCM performs best when taking a $2 \mu \mathrm{m}$ air gap into consideration.

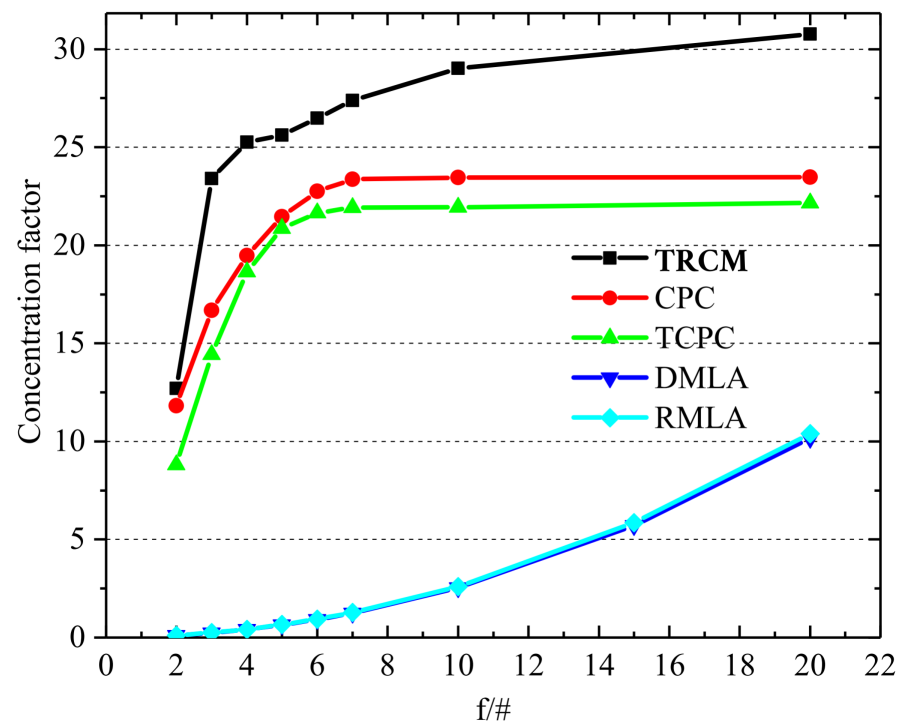

Figure 2. Concentration factors of reflective-based structures with $2 \mu \mathrm{m}$ air gap and MLAs at its focal plane (1.035 mm) simulated by Zemax. We simulated energy concentrated by the micro concentrators within a $30 \mu$ m diameter active area, as a function of the f-number. Reference wavelength: $655 \mathrm{~nm}$.

\section{EXPERIMENTS OF HIGH RESOLUTION INTENSITY AND DEPTH AND TRANSIENT RECONSTRUCTION}

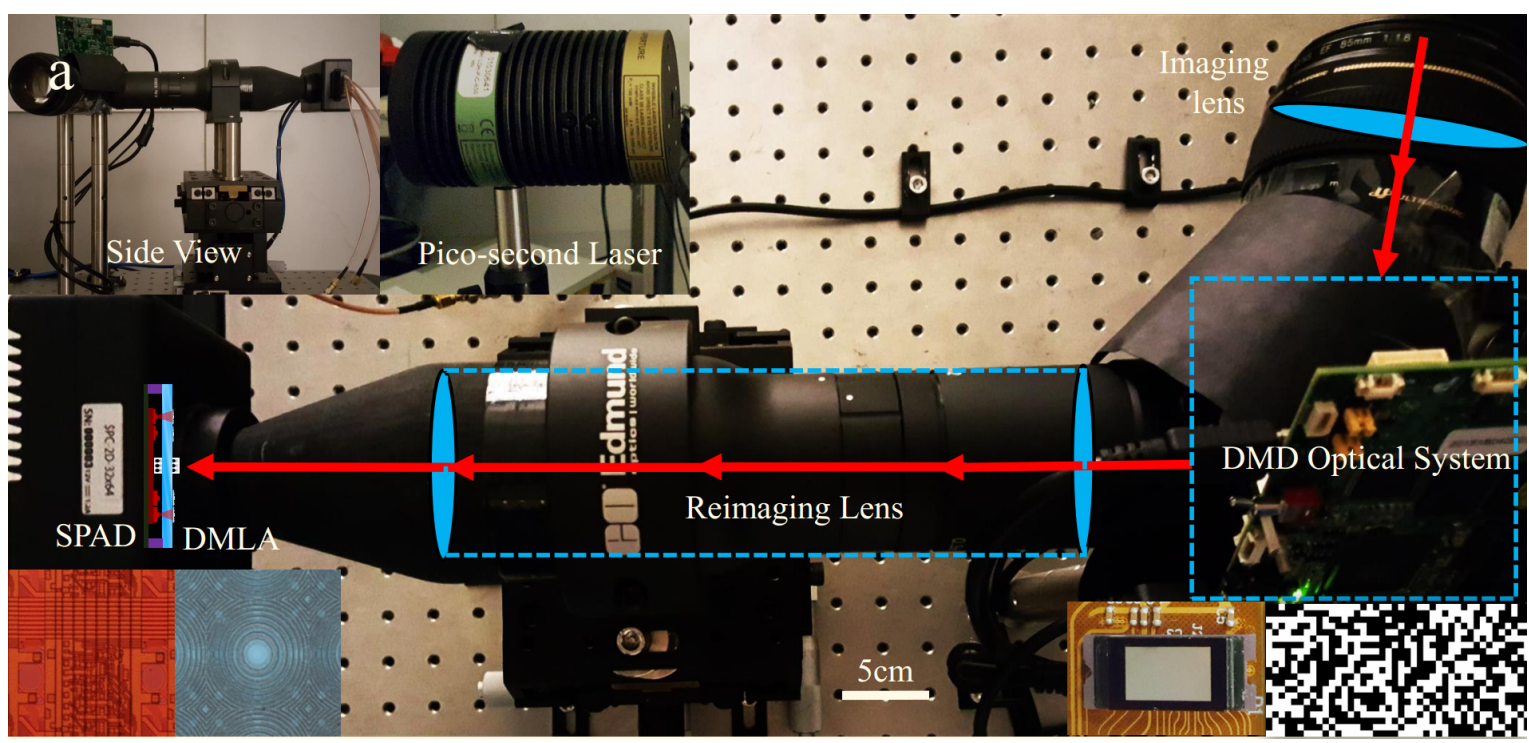

Figure 3. Optical Design and prototype of CS SPAD camera: We use a picosecond laser as the light source while the DMD optical system generate random patterns at the co-focal plane of the imaging and reimaging system. The SPAD array then receives the modulated light concentrated by the microlens array.

Figure 3a illustrates the CS-based imaging system that employs a picosecond laser light source, a programmable spatial modulator, and a SPAD array for detection. The end-to-end imaging system employs a 
similar setup with a DMLA, a DMD, and a re-imaging system that is replaced by a DOE placed in the front focal plane (set as the pupil) of the imaging lens.

\subsection{Illumination and detection}

We use a $64 \times 32$ SPAD array (MPD-SPC3) as the single photon sensitive detector. The picosecond laser source (PicoQuant LDH P-650), with the center wavelength of $655 \mathrm{~nm}$ and the maximum peak power of $360 \mathrm{~mW}$ (pulse energy $40 \mathrm{pJ}$ ), is operated at around $80 \mathrm{ps}$ pulse duration with $50 \mathrm{MHz}$ repetition rate. For CS setup, the SPAD array is operated in TCSPC mode with 20 ps shift per circle and 830 ps gate width. We set the hardware integration time (physical integration time of SPAD array) as $52 \mu$ s and hardware binning (number of frames that are summed-up before stored in the computer memory) as 1,280. The total data sent is around 3.3 million pulses per frame under one pattern. During the capturing process, a maximum $\sim 40-60$ photons per pixel can be received. Alongside the sensor, the embedded FPGA generated a $50 \mathrm{MHz}$ synchronization signal to trigger the picosecond laser. The laser beam is then scattered by a diffuser and concentrated by a biconvex lens with $30 \mathrm{~mm}$ focal length to illuminate the scene for depth or transient imaging. The capturing process, including background calibration, lasts around 9 minutes. By changing a laser source with $40 \mathrm{~nJ}$ or higher pulse energy and $450 \mathrm{~nm}$ center wavelength, the capturing process can be finished within $1 \mathrm{~s}$ theoretically. With respect to the end-to-end setup, the capturing process is simplified to a single-shot process with the laser synchronized, and the capturing process takes around 9.8 seconds.

\subsection{Results}

We present the results (2 $\pi$ DMLA) reconstructed using CS-based method. Note, that most results are implemented on the setup proposed in our recent work. ${ }^{7}$ Figure 5 shows the result of a reconstructed high-resolution image. As we can observe that the details of the characters and apple are faithfully reconstructed.
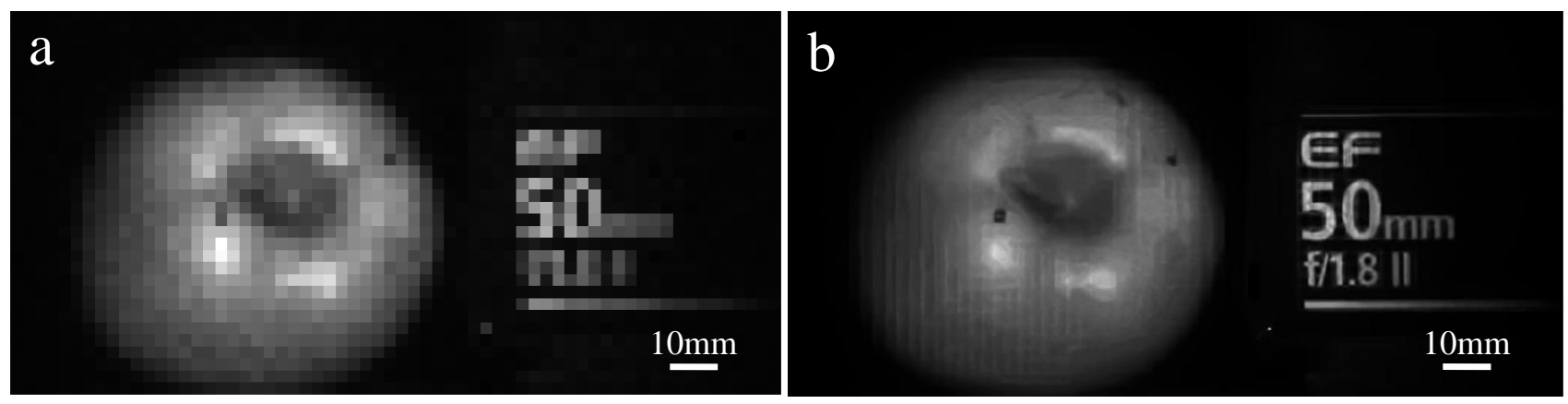

Figure 4. Result of intensity resolution improvement. (a) Background noised removed raw data modulated by the first random pattern. (b) Reconstructed high-resolution result.
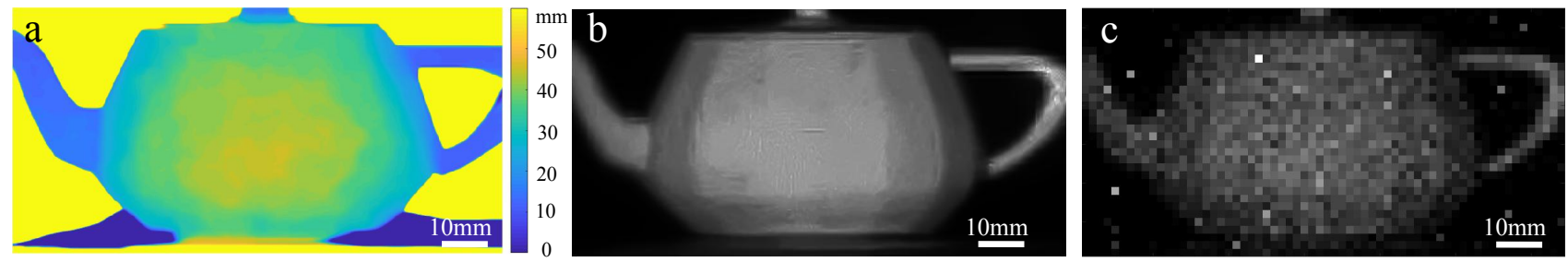

Figure 5. Result of depth resolution improvement. (a) Reconstructed high-resolution depth image. (b) Reconstructed high-resolution intensity image. (c) Low resolution averaged capture.

Figure 6 shows a re-creation of an iconic scene for light-in-flight imaging. A convex lens (50.8 mm diameter, $75 \mathrm{~mm}$ focal length) focuses a short pulse of light into a point. To visualize the light propagation, a diffusing screen lined up with the optical axis of the lens, showing a $2 \mathrm{D}$ cross-section of the $3 \mathrm{D}$ light cone (see lower right of Figure 6). 

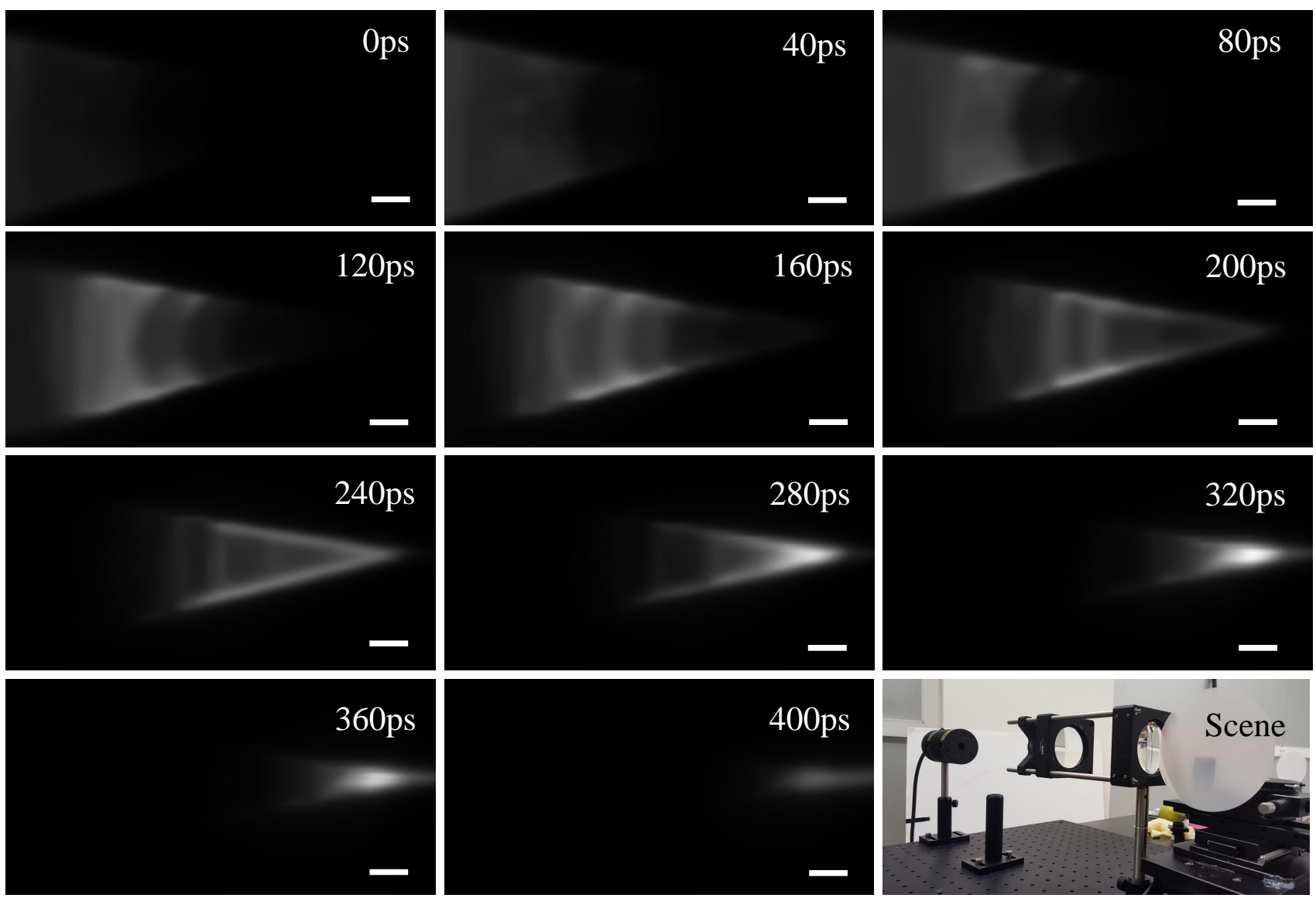

Figure 6. Result of transient frames' resolution improvement.Light concentrated by a lens We here present one frame of every two frames. Scale bar:10mm

\section{CONCLUSION}

We have summarized the optical coding solution for designing a compressive SPAD array camera with high light efficiency. This is achieved by prototyping a customized microlens array in front of the SPAD array, leading to a drastic improvement in sensor fill-factor. Further, we investigate the scenario of designing the anti-aliasing filter in an end-to-end manner, to achieve an optimal sampling for the super-resolution task. Incorporating these optical coding schemes, and combined with a compressive sensing scenario, we have resolved high-resolution 3D and transient imaging through optimization in a post-processing step. We envision our optical coding design be generalized to alternative sensing applications where fill-factor issue and resolution issue may be problematic.

\section{ACKNOWLEDGMENTS}

This project is supported by King Abdullah University of Science and Technology (KAUST) baseline funding.

\section{REFERENCES}

[1] Gariepy, G., Krstajić, N., Henderson, R., Li, C., Thomson, R. R., Buller, G. S., Heshmat, B., Raskar, R., Leach, J., and Faccio, D., "Single-photon sensitive light-in-fight imaging," Nature Communications 6 (2015).

[2] Shin, D., Xu, F., Venkatraman, D., Lussana, R., Villa, F., Zappa, F., Goyal, V. K., Wong, F. N., and Shapiro, J. H., "Photon-efficient imaging with a single-photon camera," Nature Communications 7 (2016).

[3] OToole, M., Heide, F., Lindell, D., Zang, K., Diamond, S., and Wetzstein, G., "Reconstructing transient images from single-photon sensors," Proc. IEEE CVPR (2017). 
[4] Richardson, J., Walker, R., Grant, L., Stoppa, D., Borghetti, F., Charbon, E., Gersbach, M., and Henderson, R. K., "A $32 \times 32$ 50ps resolution 10 bit time to digital converter array in $130 \mathrm{~nm}$ cmos for time correlated imaging," in [Proc. CICC], 77-80, IEEE (2009).

[5] Niclass, C., Rochas, A., Besse, P.-A., and Charbon, E., "Design and characterization of a cmos 3-d image sensor based on single photon avalanche diodes," IEEE Journal of Solid-State Circuits 40(9), 1847-1854 (2005).

[6] Guerrieri, F., Tisa, S., Tosi, A., and Zappa, F., "Two-dimensional spad imaging camera for photon counting," IEEE Photonics Journal 2(5), 759-774 (2010).

[7] Sun, Q., Dun, X., Peng, Y., and Heidrich, W., "Depth and transient imaging with compressive spad array cameras," Proc. IEEE CVPR , 273-282, IEEE (2018).

[8] Intermite, G., Warburton, R., McCarthy, A., Ren, X., Villa, F., Waddie, A., Taghizadeh, M., Zou, Y., Zappa, F., Tosi, A., et al., "Enhancing the fill-factor of cmos spad arrays using microlens integration," in [Photon Counting Applications 2015], 9504, 95040J, International Society for Optics and Photonics (2015).

[9] Pavia, J. M., Wolf, M., and Charbon, E., "Measurement and modeling of microlenses fabricated on singlephoton avalanche diode arrays for fill factor recovery," Optics express 22(4), 4202-4213 (2014).

[10] Intermite, G., McCarthy, A., Warburton, R. E., Ren, X., Villa, F., Lussana, R., Waddie, A. J., Taghizadeh, M. R., Tosi, A., Zappa, F., et al., "Fill-factor improvement of si cmos single-photon avalanche diode detector arrays by integration of diffractive microlens arrays," Optics Express 23(26), 33777-33791 (2015).

[11] Donati, S., Martini, G., and Norgia, M., "Microconcentrators to recover fill-factor in image photodetectors with pixel on-board processing circuits," Optics express 15(26), 18066-18075 (2007).

[12] Chen, H., Asif, M. S., Sankaranarayanan, A. C., and Veeraraghavan, A., "Fpa-cs: Focal plane array-based compressive imaging in short-wave infrared," in [Proc. CVPR], 2358-2366, IEEE (2015).

[13] Sitzmann, V., Diamond, S., Peng, Y., Dun, X., Boyd, S., Heidrich, W., Heide, F., and Wetzstein, G., "End-to-end optimization of optics and image processing for achromatic extended depth of field and superresolution imaging," ACM Transactions on Graphics (TOG) 37(4), 114 (2018).

[14] Wu, Y., Boominathan, V., Chen, H., Sankaranarayanan, A., and Veeraraghavan, A., "Phasecam3dlearning phase masks for passive single view depth estimation," in [Computational Photography (ICCP), 2019 IEEE International Conference on], 1-8, IEEE (2019).

[15] Chang, J. and Wetzstein, G., "Deep optics for monocular depth estimation and 3d object detection," in [Proc. IEEE ICCV], (2019).

[16] Gissibl, T., Wagner, S., Sykora, J., Schmid, M., and Giessen, H., "Refractive index measurements of photoresists for three-dimensional direct laser writing," Optical Materials Express 7(7), 2293-2298 (2017).

[17] Welford, W. T. and Winston, R., "Optics of nonimaging concentrators. light and solar energy," (1978).

[18] Winston, R., Miñano, J. C., Benitez, P. G., et al., [Nonimaging optics], Elsevier (2005).

[19] Gerchberg, R. W. and Saxton, W. O., "A practical algorithm for the determination of phase from image and diffraction plane pictures," Optik 35, 237 (1972). 